$2-2011$

\title{
Mechanical Behavior of Bio-Inspired Laminated Composites
}

\author{
Liang Cheng \\ University of Delaware \\ Adam Thomas \\ University of Delaware \\ James L. Glancey \\ University of Delaware
}

Anette M. Karlsson

Cleveland State University, a.karlsson@csuohio.edu

Follow this and additional works at: https://engagedscholarship.csuohio.edu/enme_facpub

Part of the Mechanical Engineering Commons

How does access to this work benefit you? Let us know!

\section{Publisher's Statement}

NOTICE: this is the author's version of a work that was accepted for publication in Composites Part A - Applied Science and Manufacturing. Changes resulting from the publishing process, such as peer review, editing, corrections, structural formatting, and other quality control mechanisms may not be reflected in this document. Changes may have been made to this work since it was submitted for publication. A definitive version was subsequently published in Composites Part A - Applied Science and Manufacturing, 42, 2, (02-01-2011); 10.1016/ j.compositesa.2010.11.009

\section{Original Citation}

Cheng, L., Thomas, A., Glancey, J. L., 2011, "Mechanical Behavior of Bio-Inspired Laminated Composites," Composites Part A, 42(2) pp. 211-220.

This Article is brought to you for free and open access by the Mechanical Engineering Department at EngagedScholarship@CSU. It has been accepted for inclusion in Mechanical Engineering Faculty Publications by an authorized administrator of EngagedScholarship@CSU. For more information, please contact library.es@csuohio.edu. 


\title{
Mechanical behavior of bio-inspired laminated composites
}

\author{
Liang Cheng, Adam Thomas, James L. Glancey, Anette M. Karlsson* \\ Department of Mechanical Engineering, University of Delaware, Newark, DE 19716, USA
}

\section{Introduction}

The hard exoskeletons of many arthropods, such as Homarus americanus (American lobster), Callinectes sapidus (Atlantic blue crab) and Popillia japonica (Japanese beetle), often demonstrate outstanding structural properties with multi-functional capabilities, including supporting the body weight, filtering chemicals and resisting external loads $[1,2]$. The primary components used by nature to build various exoskeletons include the chitin, proteins, water and/or minerals $[3,4]$.

Consequently, the intrinsic complex hierarchical structures of various exoskeletons have received significant attention recently, motivated by their potential to achieve excellent mechanical property and versatility for the exoskeletons [1-9]. A number of species, ranging from crustaceans (such as crabs, lobsters, and crayfish) to ancient species (such as "armored fish") have been investigated both theoretically and experimentally to explore the relationship between the microstructure and the mechanical behavior [1-10]. Interestingly, some general similarities prevail in the structural morphology across a wide range of species. A typical arthropod exoskeleton consists of several multi-layer regions (e.g., exocuticle, mesocuticle and endocuticle in the exoskeleton of a typical beetle) with load-bearing capability, see Fig. $1[1-5,8,11-13]$. Those regions are primarily built up with high stiffness chitin microfibers (Young's modulus can be as high as $100 \mathrm{GPa}$, diameter $2-3 \mathrm{~nm}$

\footnotetext{
* Corresponding author. Tel.: +1 302831 6437; fax: +1 3028313619. E-mail address: karlsson@udel.edu (A.M. Karlsson).
}

[4,14-16]) and associated proteins, as well as supplementary materials. Typically, bundles of chitin microfibers are assembled into macrofibrils with a surrounding protein matrix in crustaceans. This composite structure can be easily observed under an electron microscope $[3,7,17]$. The macro- and micro-fibrils usually align with each other to form parallel layers, which are further organized as stacks with distinctive orientations and patterns to form the exoskeletons (Fig. 1) [1-11,18].

Even though many bio-inspired, man-made structures exist, a very limited amount of research has been conducted which attempts to apply the design principles in the arthropod exoskeletons into the design and manufacturing of practical materials and structures. Apichattrabrut [19] replicated a limited set of helicoidal laminated composite structures. The resulting structures were reported to demonstrate improved mechanical performance over the conventional unidirectional and cross-ply composites. However, this work only considered a limited set of geometries. Consequently, we here consider multiple structures along with investigating the residual strength after the onset of initial failure.

Thus, with the ultimate purpose of exploring and designing advanced engineering materials and structures inspired by nature, the objectives of this study are: (1) adopt representative structural morphology observed in arthropod exoskeletons (the helicoidal structure in the exoskeletons from $\mathrm{H}$. americanus, $\mathrm{C}$. sapidus and $P$. japonica) $[1,2]$ in the design of nature-inspired composites; (2) manufacture the composites with commonly used engineering materials: (3) investigate the mechanical responses of the resulting bio-inspired structures from multiple perspectives, through both experimental characterization and theoretical analysis; and (4) 


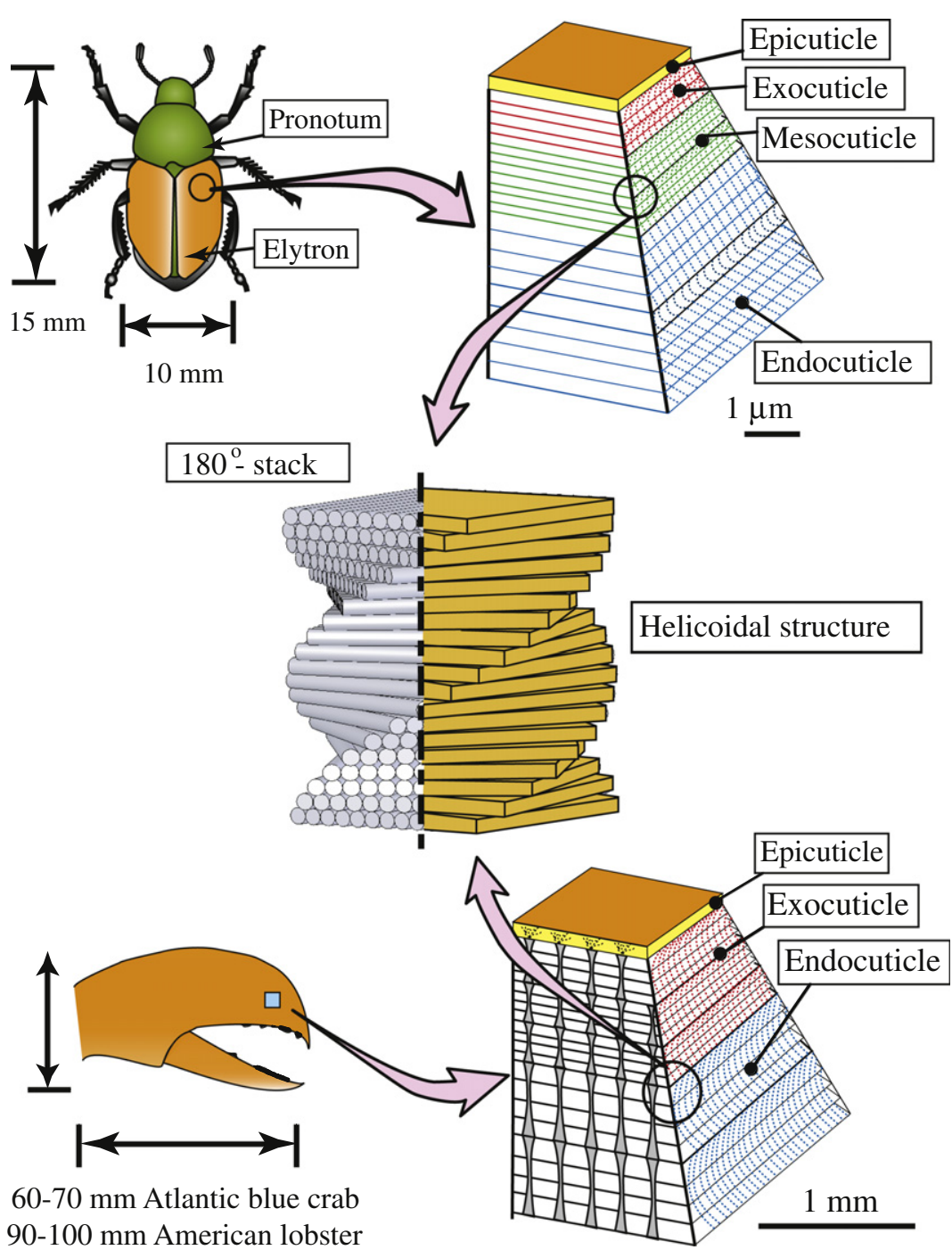

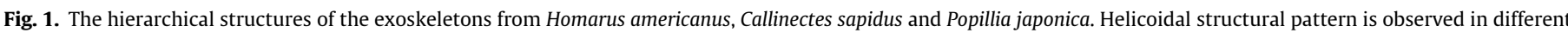
regions of all the exoskeletons $[1,2]$.

compare the results to identify and summarize advantageous design strategies to formulate guidance for developing advanced synthetic materials.

\section{Materials and methods}

\subsection{Helicoidal structure}

Helicoidal structure, also termed "Bouligand structure" after its discovery by Bouligan [17] is one of the unique and prevailing patterns observed in exoskeletons in a large number of species of the arthropod phylum $[3,7,11,12,20-23]$, including crustaceans (e.g., $C$. sapidus and $C$. sapidus) and insects (e.g., P. japonica) [1,2]. The structure is characterized by the parallel chitin-protein layers stacking successively on each other, with each uni-directional layer rotating by a small angle about its normal direction relative to the adjacent layer, as shown in Fig. 1.

Our previous studies [1,2] have shown that a helicoidal structure with a moderately large number of layers results in a high level of in-plane isotropy, providing isotropic structural response in the loading plane. In addition, since the gradual rotation of successively stacked layers effectively smoothens the stiffness transition cross each interface between adjacent layers compared to tradi- tional cross-ply laminated composites, the helicoidal structure appears to possess relatively high interfacial strength $[1,2]$.

All together, the helicoidal structure shows great potential for providing high stiffness, high strength and durable structures. Thus, this study is focused on investigating selected key features of helicoidal structures.

\subsection{Materials, design and manufacture}

\subsubsection{Materials and processing}

A commonly used glass fiber reinforced composite was selected as the model material system for exploring the bio-inspired composite. The material used for all manufacturing and processing in this study is unidirectional S2-glass (Zentron High Strength fiber) epoxy prepreg, DA409U/S2-glass (APCM, Plainfield, CT, USA). The physical and mechanical properties of this material from a representative batch are summarized in Table 1, based on independent standard tests (ASTM D790 [24], ASTM D2344 [25]) under our lab conditions (described later). Throughout the development and test process, multiple batches of material were used. The same standard test protocols were followed for each individual batch prior to use in order to extract relevant baseline material properties (as in Table 1), and to eliminate potential effects of cross-batch 
Table 1

Mechanical properties of DA 409U/S2-glass from a source material batch (\#2). Data provided by original manufacturer are marked with an asterisk $\left({ }^{*}\right)$, others are from our independent standard test. $E_{1}$ and $E_{2}$ are Young's moduli along the direction of the fiber and orthogonal to the fiber, respectively; $G_{12}$ and $G_{23}$ are the shear moduli; $v_{12}$ and $v_{23}$ are Poisson's ratios. $\sigma_{1}$ is the flexural strength (ASTM D790) and $F^{\text {sbs }}$ is the short-beam shear strength (ASTM D2344). $\rho$ is the material density.

\begin{tabular}{lc}
\hline$E_{1}(\mathrm{GPa})$ & 47.7 \\
$E_{2}(\mathrm{GPa})$ & 4.77 \\
$G_{12}(\mathrm{GPa})$ & 7.55 \\
$G_{23}(\mathrm{GPa})$ & 5.06 \\
$v_{12}$ & 0.28 \\
$v_{23}$ & 0.45 \\
$\sigma_{1}(\mathrm{GPa})$ & 0.89 \\
$F^{\text {sbs }}(\mathrm{GPa})$ & 0.57 \\
$\rho^{*}\left(\mathrm{~kg} / \mathrm{m}^{2}\right)$ & 0.41 \\
Thickness per layer* $(\mathrm{mm})$ & 0.28 \\
\hline
\end{tabular}

material property variations. In addition, all the analysis and test results of each sample were consistently non-dimensionalized with respect to the material properties of its parent material batch before further presentation, analysis and comparison.

The prepreg rolls were stored in a contamination free container under $-18{ }^{\circ} \mathrm{C}\left(0{ }^{\circ} \mathrm{F}\right)$ before processing. Prior to manufacturing, each roll was removed from cold storage at least $20 \mathrm{~h}$ prior to use to allow for stabilization at room temperature, and wrapped to prevent moisture from condensing on the prepreg.

Prepreg rolls $\left(0.3 \times 110 \mathrm{~m}^{2}(12\right.$ in. $\times 120$ yards $\left.)\right)$ were cut into square pieces $\left(0.3 \times 0.3 \mathrm{~m}^{2}\left(12 \times 12\right.\right.$ in. $\left.\left.^{2}\right)\right)$ before being laid up to construct laminated composite plates. All laminates were stacked with 24 plies using selected stacking sequences corresponding to the selected structural configurations (described below). The stacked prepreg laminates were then cured using the vacuum bagging method. The sequence for the vacuum bagging system is illustrated in Fig. 2A. Peel plies (Bleeder lease BC, AIRTECH

A
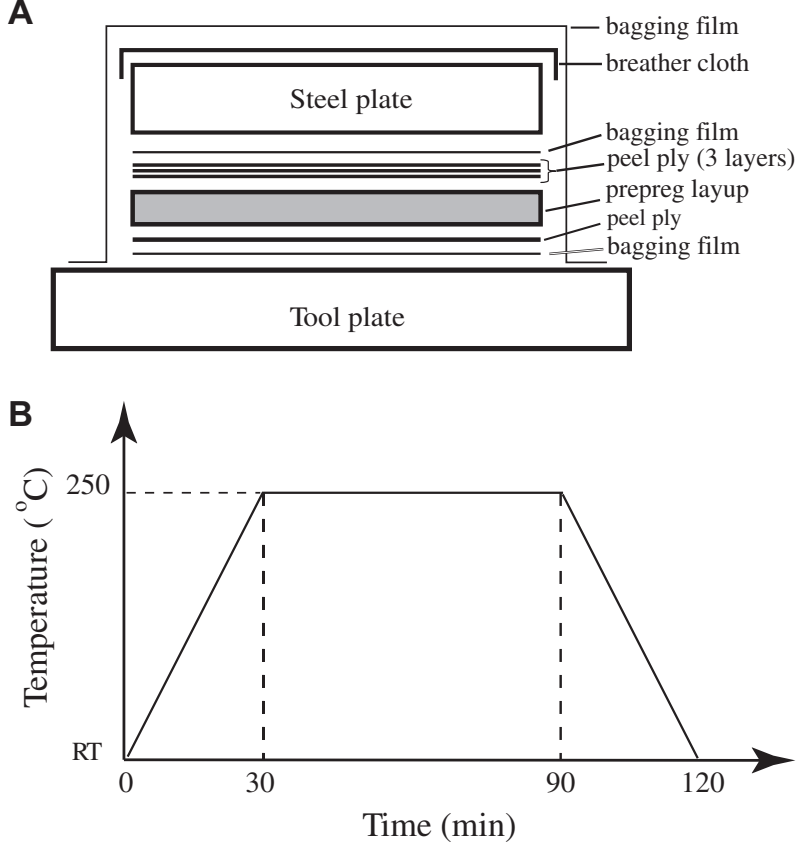

Fig. 2. (A) Schematic diagram of the manufacturing of the composite structures; (B) typical cure cycle used in composite manufacturing.
International Inc., Huntington Beach, CA, USA) were attached on both sides of the stacked prepreg to render a smooth finish on the laminates and protect the laminates from potential contamination. Breather cloth (Airweave N-10 breather cloth, AIRTECH International Inc., Huntington Beach, CA, USA) was put on top of the steel plate to prevent cutting or penetration by the steel plate on the outside bagging film (Wrightlon 7400 bagging film, AIRTECH International Inc., Huntington Beach, CA, USA). The bagging film was attached firmly to the tool plate using tacky tape (SM 5126 Tacky tape, Schnee-Morehead Inc., Irving, Texas, USA). Vacuum was then supplied to the system in the oven before curing and maintained during the entire curing process. The oven was programmed to implement the designed cure cycle for the prepreg, as shown in Fig. 2B. The whole lay-up system, including the laminate and all the other facilities described above, was finally retrieved from the oven after the curing cycle was finished and cooled to room temperature.

\subsubsection{Mid-plane symmetry}

Mid-plane symmetry is generally preferred in practical design and manufacturing of laminated composites, since it simplifies the design and manufacturing process, and eliminates warping induced during cure (e.g., causes the $\boldsymbol{B}$ matrix defined in Appendix A to vanish).

However, the nature designed helicoidal structure, as in the exoskeletons from $H$. americanus and $C$. sapidus, has an anti-symmetrical stacking sequence, resulting in a laminate with coupled in-plane and out-of-plane behavior [1]. Thus, when this structure is replicated using conventional composite materials and manufacturing processes, residual stresses induced during the laminate curing process result in warping of the manufactured laminate. The resulting curvature is determined by the component's lamina material properties, including stiffness, thermal expansion coefficient and Poisson's ratio, as well as the laminate stacking sequence [26]. Further, the anti-symmetrical structure causes unbalanced and coupled stress and strain distribution, contributing to a complex and mostly undesired structural behavior, which may compromise the structural integrity and stability. As a result, although the bio-inspired composites inherit potential mechanical benefit from the exoskeleton, the anti-symmetry limits their practical usefulness. (In nature, these issues are not of concerns, since "manufacturing" occur at ambient conditions.)

Consequently, in addition to a direct replication of the natural helicoidal structure in the bio-composite design, two variations were designed (Table 2) as described in the following section. Both designs intend to solve the unsymmetrical problem by enforcing a mid-plane symmetry while maintaining the gradual ply rotation pattern in the laminate stacking sequence. The resulting laminates remained flat after manufacturing.

\subsubsection{Bio-composite design schemes}

In this study, four lay-up sequences were designed and manufactured; each with 24 plies (Table 2). The four designs included one baseline structure and three bio-inspired structures:

(1) a "baseline" structure (BL), with mid-plane symmetry commonly used in industry as a quasi-isotropic structure, with stacking sequence $\left[0 /-45^{\circ} / 45^{\circ} / 90\right]_{3 s}$;

(2) a "single helicoidal" structure ( $\mathrm{SH})$, with a stacking sequence directly replicated from the nature designed helicoidal structure described earlier. A representative " $180^{\circ}$ stack" with 24 plies completes an accumulative rotation of $180^{\circ}$ about the normal direction of the uni-directional layers, thus a $7.8^{\circ}$ rotation per ply is needed. The stacking sequence is [0/ $7.8^{\circ} \% / 180^{\circ}$ ] thus mid-plane symmetry is not satisfied (in fact, the structure is anti-symmetric); 
Table 2

Investigated laminate structures and their stacking sequences.

\begin{tabular}{llll}
\hline $\begin{array}{l}\text { Structure } \\
\text { designation }\end{array}$ & Specification & $\begin{array}{l}\text { Number of } \\
\text { lamina }\end{array}$ & $\begin{array}{l}\text { Stacking } \\
\text { sequence }\end{array}$ \\
\hline BL & Baseline & 24 & {$\left[0 /-45^{\circ} / 45^{\circ} / 90^{\circ}\right]_{3 \mathrm{~s}}$} \\
SH & Single helicoidal & 24 & {$\left[0 / 7.8^{\circ} / \ldots / 180^{\circ}\right]$} \\
DH & Double helicoidal & 24 & {$\left[0 / 16.4^{\circ} \% / 180^{\circ}\right]_{s}$} \\
SHMS & Single helicoidal & 24 & {$\left[0 / 7.8^{\circ} / \ldots / 85.8^{\circ}\right]_{\mathrm{s}}$} \\
& mid-plane symmetric & & \\
\hline
\end{tabular}

(3) a "double helicoidal" laminate (DH), with both the upper and lower halves (12 plies each) completing an entire $180^{\circ}$ accumulative rotation individually, and stacked together symmetrically about their mid-plane. It enforces the midplane symmetry design but results in a larger ply rotation across the thickness, compared to Scheme 2. Its stacking sequence is $\left[0 / 16.4^{\circ} / \ldots / 180^{\circ}\right]_{s}$;

(4) a "single helicoidal mid-plane symmetric" laminate (SHMS), with its upper half (12 plies) following the same pattern as that of the single helicoidal laminate, but its lower counterpart is mirrored with respect to the mid-plane of the laminate. Therefore SHMS enforces mid-plane symmetry while retaining the smaller ply rotation as in the $\mathrm{SH}$ laminate. Its stacking sequence is $\left[0 / 7.8^{\circ} / \ldots / 85.8^{\circ}\right]_{s}$.

\subsection{Experimental investigations}

The mechanical properties of the four laminates were investigated via ASTM test protocols, including flexural stiffness and maximum flexural stress ["long beam test" (ASTM D790)], [24] and short-beam shear strength ["short beam test" (ASTM D2344)] [25]. Both tests are based on three-point bending with characteristic dimensions shown in Fig. 3.

Laminated beam samples were cut (using ACER AGS-1020-AH Hydraulic Surface Grinder, Klim Industrial, Inc., Piscataway, NJ, USA) from the manufactured laminate plates with compatible sizes in accordance with corresponding test protocols:

(1) for ASTM D790, "the specimen support span-to-thickness ratio $(L / h)$ shall be 16 (tolerance \pm 1 ) with overhanging length on each end at least $10 \%$ of the support span; specimen width $(b)$ shall not exceed 1/4 of the support span;" [24].

(2) for ASME D2344, "specimen support span-to-thickness ratio $(L / h)$ is 4 and specimen width-to-thickness ratio $(b / h)$ is 2 . The overhang on each end of the support is controlled to approximately one thickness value." [25].

Samples were cut from the center of the laminate plate, to ensure a complete lay-up and consistent thickness. Care was taken in the sample cutting process to ensure the reinforcing glass fibers on the beam outer surfaces (top and bottom) were aligned with the beam length direction.

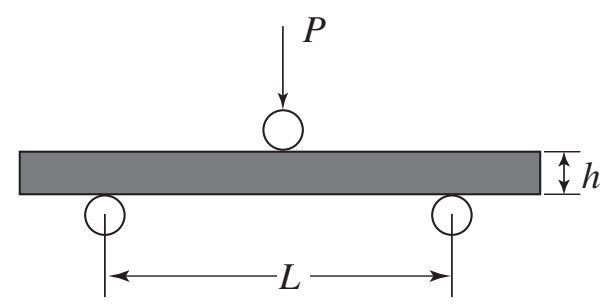

Fig. 3. Illustration of the test configuration (both ASTM D790 and ASTM D2344). $L$ is the beam supporting span, $h$ is the beam thickness and $P$ is the applied force.
Relevant geometric parameters and physical properties of the beam specimen were measured after sample preparation. The specimen size was measured at five locations along each dimension, and averaged values were used for analysis.

Composite void content for each configuration was also measured following the corresponding ASTM procedure (ASTM D2734) [27]. Representative materials were harvested from the source laminates and in the immediate proximity of the corresponding beam specimens. The void contents are presented along with the corresponding test results in the following section.

For both standard tests, an Instron 5567 Tester Frame (Norwood, MA, USA) was used. The beam specimens were loaded to failure in a three-point-bending system, with simply supported boundary conditions, Fig. 3. All the other test-related parameters (e.g., the loading rate, the loader-nose size) were chosen in accordance with the corresponding test standard.

Concurrent to the manufacturing and processing described above, a 24-ply unidirectional laminate was produced for each individual parent material batch. The unidirectional laminate was manufactured and corresponding samples were processed and tested according to the same protocols described above, and analyzed in according to methods in the following section. The material data obtained were used as the true reference material properties for each material batch (Table 1 ). Due to the nature of the unidirectional fiber reinforced composites, only the material properties along the principal direction (fiber direction) were investigated. The material properties in the directions orthogonal to the fiber orientation have limited influence of the overall structure behavior, thus general empirical value from engineering experience and the handbook "MIL-HDBK-17-1F: Composite Materials Handbook" [28] were used (e.g., Young's modulus in the directions orthogonal to the fiber can be assumed being only $1 \%$ of that along the fiber direction: $E_{2}=E_{3}=0.01 E_{1}$; and the relationship between the shear modulus for general glass fiber/epoxy composites is: $G_{23}=0.67 G_{12}$ ), and are summarized in Table 1 .

\section{Results}

\subsection{Long beam test results (ASTM D790)}

The material properties quantified from the "long beam test" (ASTM D790) are the flexural modulus $\left(E_{B}\right)$ and flexural strength $\left(\sigma_{f M}\right)[24]$ :

$E_{B}=\frac{m L^{3}}{4 b h^{3}}$

$\sigma_{f M}=\frac{3 P_{m} L}{2 b h^{2}}$,

where $h, b$ are the thickness and width of the beam respectively ( $m$ ), $L$ is the supporting span (Fig. 3 ) $(\mathrm{m}), P_{m}$ is the maximum load observed during the test $(\mathrm{N})$, and $m$ is the slope of the tangent to the initial straight-line portion of the force-deflection curve $(\mathrm{N} / \mathrm{m})$.

Due to variation in material properties between the various material batches used, and a variation in dimensions due to variability in manufacturing, the measured force $P$ and measured displacement $\delta$ are normalized according to:

$\widehat{P}=\frac{P}{2 E b h^{3} / 3 L}$,

$\hat{\delta}=\frac{\delta}{h}$,

where $\widehat{P}$ and $\hat{\delta}$ are the normalized force and displacement, respectively. The normalized force, $\widehat{P}$, as a function of the normalized displacement, $\hat{\delta}$, for the four configurations investigated are displayed in Fig. 4. 

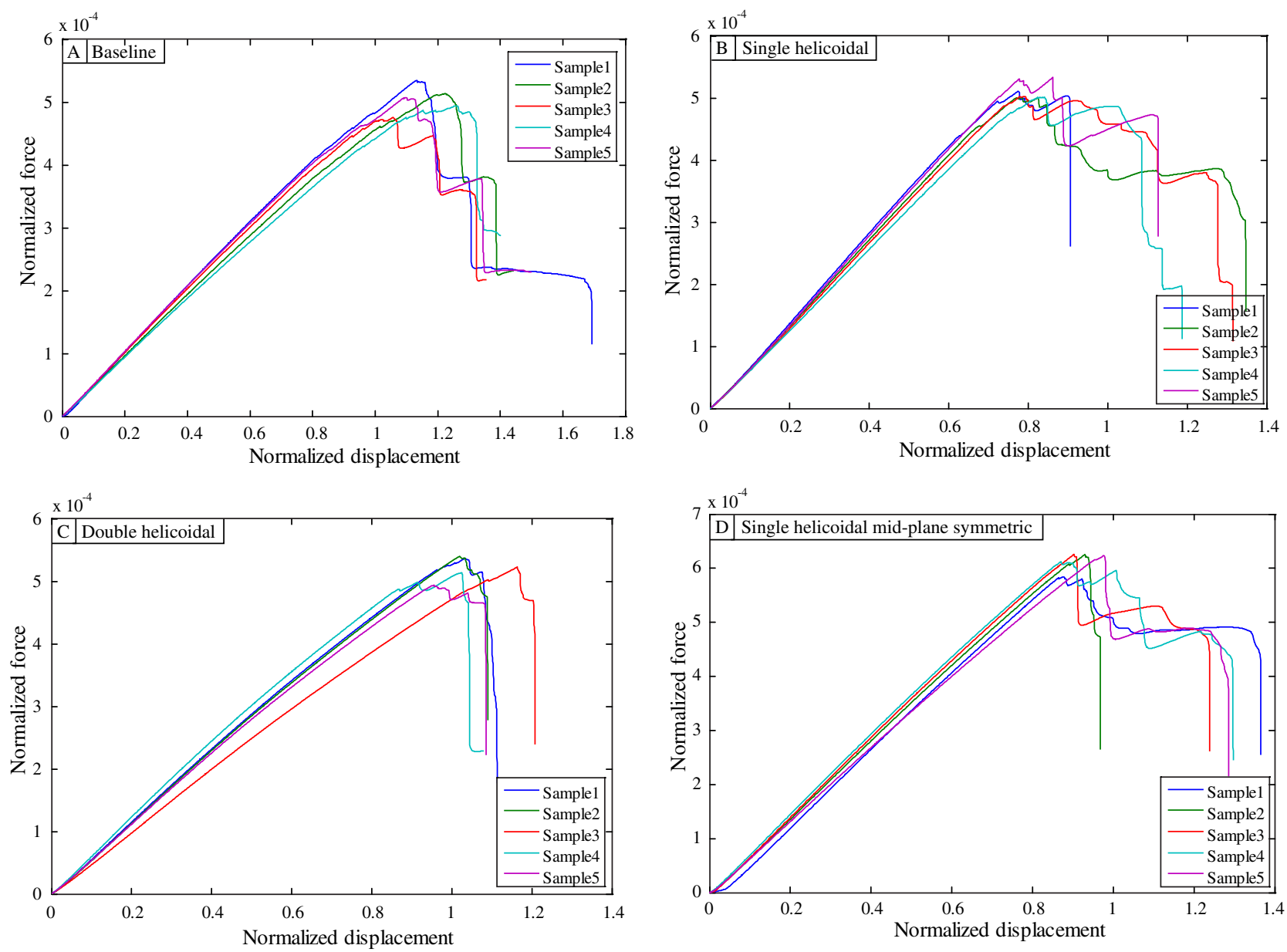

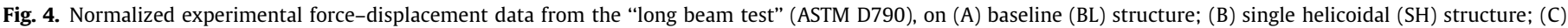
double helicoidal (DH) structure; (D) single helicoidal mid-plane symmetrical (SHMS) structure.

The flexural stiffness and flexure strength are determined according to ASTM D790 Eqs. (1) and (2). The averaged results (with deviations) are reported after normalization with respect to the corresponding material properties of each specimen's source material batch, as summarized in Table 3.

For comparison, theoretical analysis was performed to estimate laminate material properties based on the classic laminated composites theory $[29,30]$. The equivalent flexural modulus for a laminate, $E_{B E}$, can be predicted theoretically given the parent batch material properties and laminate stacking configuration (Tables 1 and 2). The corresponding components $\left(D_{i j}\right)$ in the laminate flexural stiffness matrix, $\boldsymbol{D}$, can first be calculated based on the given information (Appendix A) and the equivalent flexural modulus is given by:
$E_{B E}=\frac{12 D_{11}}{h^{3}}$,

where $D_{11}$ is the flexural stiffness component along the beam length direction. Both the experimental and theoretical results are given in Table 3 and Fig. 5.

The experimental results and the theoretical predictions show excellent agreement for three of the laminate structures (Fig. 5). For the single helicoidal (SH) structure, the experimental data deviated noticeably from the theoretical value. We believe the discrepancy is caused by the high void content in these specimens induced during manufacturing, Table 3 . The overall trend in the flexural stiffness can be seen in Fig. 5A: the flexural stiffnesses of the bioinspired helicoidal structures are all higher than the baseline (BL)

Table 3

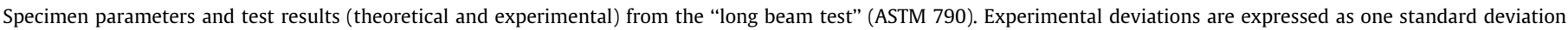
about the mean.

\begin{tabular}{|c|c|c|c|c|c|}
\hline Structure & & Baseline & Single helicoidal & Double helicoidal & Single helicoidal mid-plane symmetric \\
\hline Number of replicates tested & & 5 & 5 & 5 & 5 \\
\hline Length, $L(\mathrm{~mm})$ & & 76.00 & 76.00 & 72.00 & 71.00 \\
\hline Width, $b(\mathrm{~mm})$ & & 11.06 & 10.61 & 10.25 & 10.16 \\
\hline Thickness, $h(\mathrm{~mm})$ & & 4.84 & 5.01 & 4.52 & 4.44 \\
\hline Void content (\%) & & 1.3 & 2.2 & 0.8 & 1.0 \\
\hline \multirow[t]{2}{*}{ Normalized flexural stiffness $\left(E_{B}\right)$} & Theory [Eq. (5)] & 0.47 & 0.60 & 0.55 & 0.74 \\
\hline & Experiment [Eq. (1)] & $0.48( \pm 0.01)$ & $0.74( \pm 0.01)$ & $0.52( \pm 0.02)$ & $0.74( \pm 0.01)$ \\
\hline Normalized flexural strength $\left(\sigma_{f M}\right)$ & & $0.64( \pm 0.03)$ & $0.63( \pm 0.01)$ & $0.57( \pm 0.02)$ & $0.67( \pm 0.02)$ \\
\hline
\end{tabular}



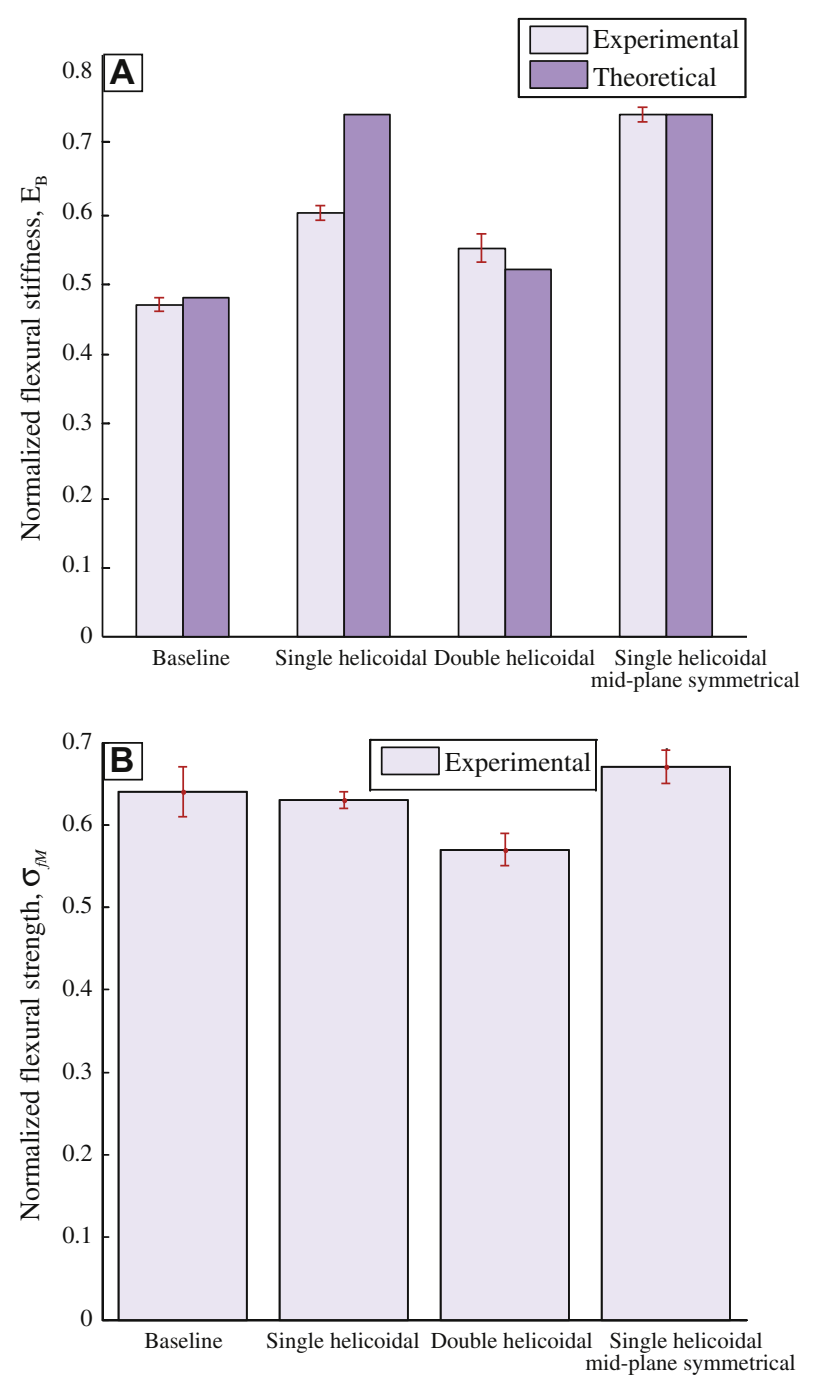

Fig. 5. Mechanical properties of the laminate beam structures using the "long beam test" (ASTM D790): (A) normalized flexural stiffness $\left(E_{B}\right)$, from both experimental result and theoretical prediction; (B) normalized flexural stress $\left(\sigma_{f M}\right)$. The error bars indicate the standard deviation of the result within each group.

structure. The flexural stiffness increase of the double helicoidal (DH) laminate is small compared to the other two bio-inspired structures. This is due to the relatively "coarse" ply rotation. The single helicoidal ( $\mathrm{SH}$ ) and single helicoidal mid-plane symmetric (SHMS) laminates, which feature a smaller ply rotation between neighboring layers, both exhibit a significant flexural stiffness increase, where theoretically a $54 \%$ relative improvement is predicted. However, as stated previously, due to the relatively large void content of the manufactured single helicoidal $(\mathrm{SH})$ laminates (Table 3), the experimental results show an increase that is lower than the prediction.

\subsection{Short beam test results (ASTM D2344)}

In the "short beam test" (ASTM D2344), short-beam strength $\left(F^{s b s}\right)$ is defined by:

$F^{s b s}=\frac{0.75 P_{m}}{b h}$,

where the parameters are the same as defined in Eqs. (1) and (2).

In order to ensure the validity and accuracy of the shear property evaluation, only the samples observed with clear interlaminar failure during the loading were analyzed accordingly. Test data from ASTM D2344, normalized in a similar manner as for ASTM D790, are shown in Fig. 6 and a summary of these results, are presented in Table 4 and Fig. 7.

ASTM D2344 does not provide a method to determine the transverse shear modulus, $G_{T}$, since the test is designed to determine the "short-beam strength" only. However, the small span-to-thickness ratio of the specimen increases the contribution of the transverse shear to beam deformation. Therefore, we will use the results from this particular test to assess the transverse shear modulus $G_{T}$, according to the following.

"Timoshenko beam theory" incorporates the transverse deformation due to transverse shear deformation into the total beam deflection [31]. Assuming a constant transverse shear stress distribution through the beam thickness, the theory gives a simplified but adequate approximation for the mechanism of short beam deformation. The deflection at the beam center span, $\omega$, is given by [31]:

$\left.\omega=\& \frac{L^{3}}{4 b h^{3}} \frac{1}{E_{B}}+\frac{3 L}{10 b h} \frac{1}{G_{T}}\right),($

where $P$ is the applied force and $E_{B}$ is the flexural stiffness (in this study obtained through the "long beam test", ASTM D790). Therefore, the transverse shear modulus $\left(G_{T}\right)$ can be obtained from the experimental data:

$G_{T}=\frac{\frac{3 L}{10 b h}}{\frac{\omega}{P}-\frac{L^{3}}{4 b h^{3}} \frac{1}{E_{B}}}$,

where $\omega / P$ is the apparent beam compliance $(\mathrm{m} / \mathrm{N})$, which is the inverse of the slope of the tangent to the initial linear portion of the force-deflection curve. It corresponds to the linear elastic response at the early stage of the loading, before the onset of non-linear behavior.

Alternatively, theoretical prediction of the equivalent transverse shear modulus $\left(G_{T E}\right)$ of the laminates can be established based on classic laminate composite theory $[29,30]$, given the parent material properties and laminate stacking configuration (Tables 1 and 2):

$G_{T E}=\frac{A_{55}}{h}$,

where $A_{55}$ is the corresponding transverse shear component in the laminate extensional stiffness matrix $\boldsymbol{A}$ (Appendix A).

The experimentally-based (using "Timoshenko beam theory", Eq. (8)) and the theoretically-based (using classic laminate composite theory, Eq. (9)) transverse shear modulus predictions are summarized in Table 4 and Fig. 7A. The methods show good agreement, verifying the validity of Timoshenko beam theory in describing the beam deformation mechanism for these specimens.

The results of the transverse shear modulus, $G_{T}$, and the shortbeam shear strength, $F^{s b s}$, from the bio-inspired composites did not show noticeable improvement over those from the baseline composite (Fig. 7). It may be attributed to the fact that transverse shear modulus, as well as transverse shear strength, of a typical fiber-reinforced laminate is more dependent on the matrix properties rather than that of the properties and orientation of the reinforcing fibers. Consequently, unlike the case of the flexural stiffness investigated in the "short beam test" (ASTM 790), the stacking sequence serves a secondary role in shaping the laminate's transverse shear property.

\section{Discussion}

Excessive load and deformation in a composite structure will lead to damage and ultimately failure, resulting in service-life 

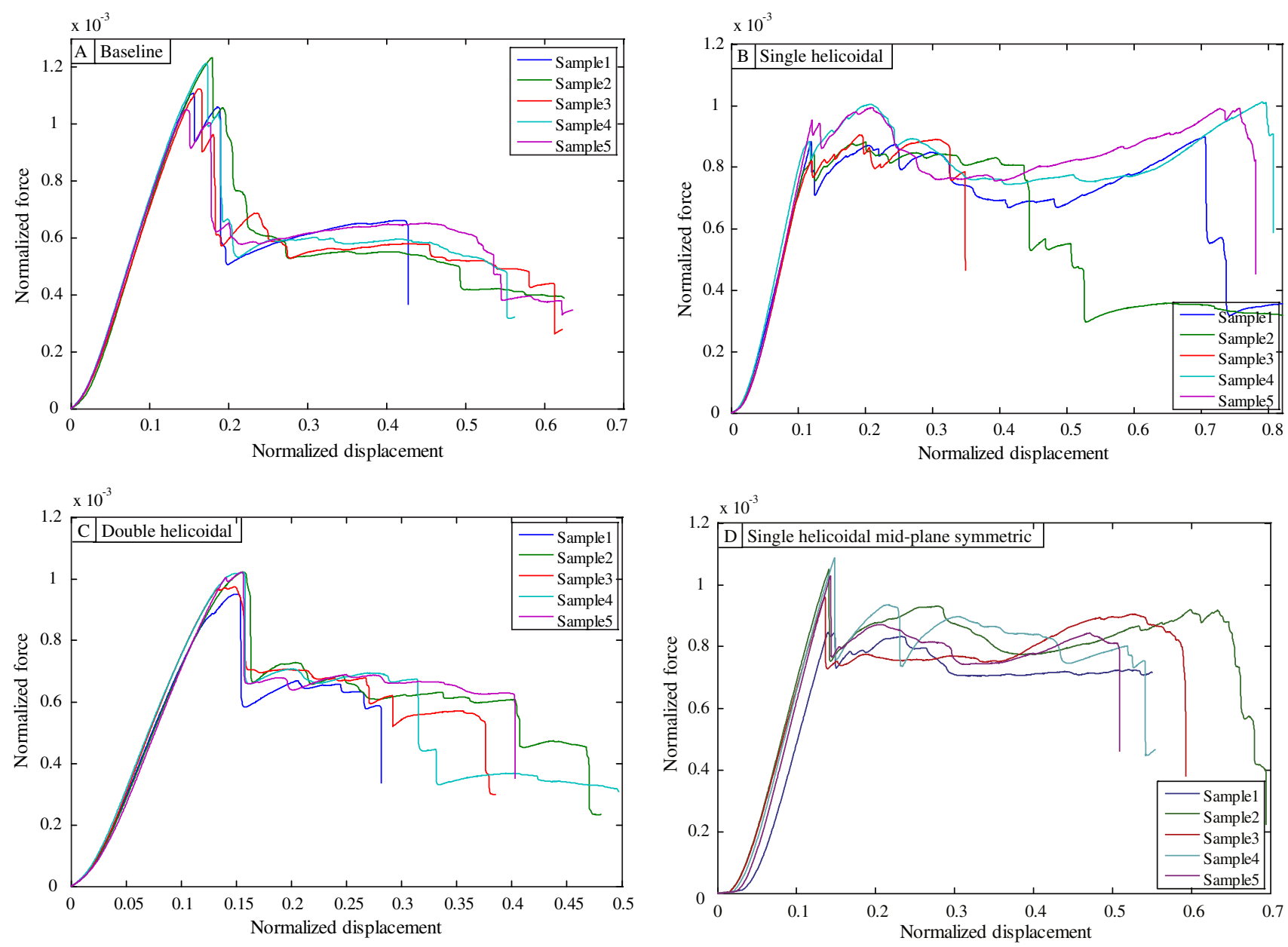

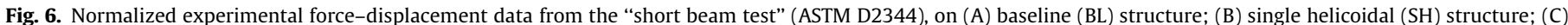
double helicoidal (DH) structure; (D) single helicoidal mid-plane symmetrical (SHMS) structure.

Table 4

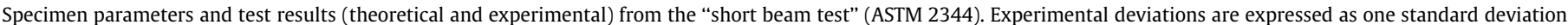
about the mean.

\begin{tabular}{|c|c|c|c|c|c|}
\hline Structure & & Baseline & Single helicoidal & Double helicoidal & Single helicoidal mid-plane symmetric \\
\hline Number of replicates tested & & 5 & 5 & 5 & 5 \\
\hline Length, $L(\mathrm{~mm})$ & & 19.00 & 19.50 & 18.30 & 17.50 \\
\hline Width, $b(\mathrm{~mm})$ & & 11.01 & 8.29 & 10.43 & 10.09 \\
\hline Thickness, $h(\mathrm{~mm})$ & & 4.92 & 5.11 & 4.52 & 4.39 \\
\hline Void content $(\%)$ & & 1.3 & 2.2 & 0.8 & 1.0 \\
\hline \multirow[t]{2}{*}{ Normalized transverse shear modulus $\left(G_{T}\right)$} & Theory [Eq. (9)] & 0.84 & 0.83 & 0.82 & 0.83 \\
\hline & Experiment [Eq. (8)] & $0.83( \pm 0.04)$ & $0.87( \pm 0.05)$ & $0.92( \pm 0.07)$ & $0.86( \pm 0.02)$ \\
\hline Normalized short-beam shear strength $\left(F^{s b s}\right)$ & & $0.76( \pm 0.02)$ & $0.80( \pm 0.04)$ & $0.76( \pm 0.03)$ & $0.76( \pm 0.07)$ \\
\hline
\end{tabular}

degradation. The onset of damage is usually characterized by the degradation of structural stiffness and load-bearing capability. In composite structures, multiple mechanisms are involved in the damage initiation and evolution, including fiber breakage, matrix cracking, fiber-matrix debonding and delamination [32-34]. Delamination is generally the most common and serious mode of failure. Due to their complicated damage evolution, composite structures usually demonstrate a progressive degradation of material stiffness and strength, instead of failing catastrophically upon initial damage onset $[35,36]$. As a result, after damage initiation, the structure usually can still function with a residual load-carrying capacity until ultimate failure. Thus, evaluating the post-damage behavior of a composite can reveal important information.
The "short beam test" (ASTM D2344) described in the previous section will be used to study the residual strength of the investigated laminates under a transverse quasi-static load. The structures were continuously loaded (using displacement-controlled loading) after initial stiffness degradation until ultimate failure occurred (which is characterized by the complete loss of load-carrying capacity). Due to the small span-to-thickness ratio of the specimen required by the test protocol, the transverse shear stress is substantial in the structure compared to samples with large span-to-thickness ratio. Delamination modes (both along initial interface and branched through the thickness) were dominant during failure, but other modes were also observed (e.g., matrix crack, and fiber pull-out). 

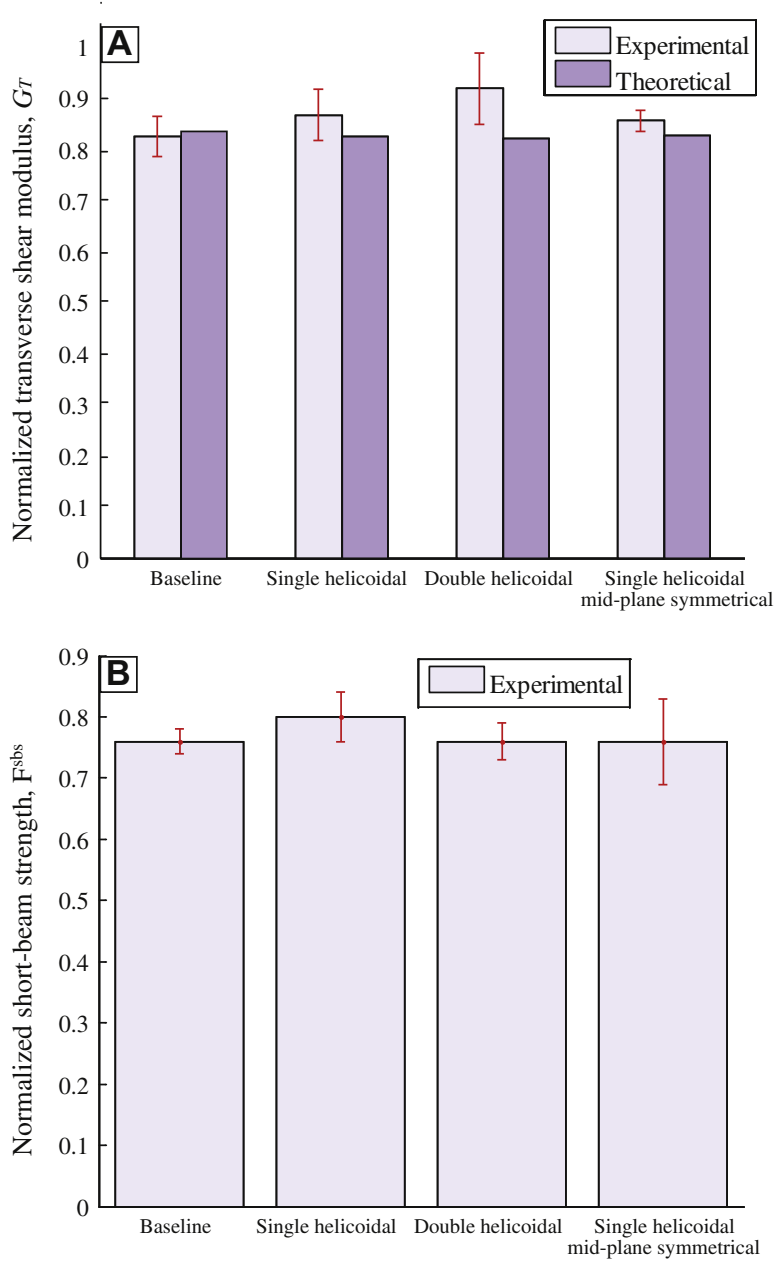

Fig. 7. Mechanical properties of the laminate beams structures using the "short beam test" (ASTM D2344): (A) normalized transverse shear modulus $\left(G_{T}\right)$, from both experimental result and theoretical prediction; (B) normalized short-beam strength $\left(F^{s b s}\right)$. The error bars indicate the standard deviation of the result within each group.

A representative force-displacement curve from a laminated composite structure subjected to three-point-bending is shown in Fig. 8. The initial response (A-B) is dominated by the linear elastic properties of the constituent materials. An abrupt drop in the force $(B-C)$ is an indication of initial damage onset (delamination initiation) in the structure. Elastic energy is absorbed by the structure in $\mathrm{A}-\mathrm{B}$ and is partly dissipated by the delamination in $\mathrm{B}-\mathrm{C}$. Upon continuous displacement-controlled loading, the delaminations grow (together with other possible failure modes) (C-D-E). Although the structural integrity is compromised, the structure can typically sustain significant load, Fig. 6, enabling it to function at a reduced load level (e.g., an airplane could make a safe emergency landing, "limp home"). The absorbed energy during this stage is dissipated as the failure modes evolve. The final failure (E-F) is characterized by the structure reaching its maximum degradation and is completely deprived of its residual load-carrying capacity.

Although the post-damage behavior of a composite structure is complex due to the involvement of multiple coupled mechanisms, the force-displacement response of the structure under threepoint-bending still reveals some characteristic structural behavior. The ultimate failure occurred approximately at the same displacement for all the configurations (Fig. 6). Thus, the residual strength indicates the post-damage energy-absorption capability for a given

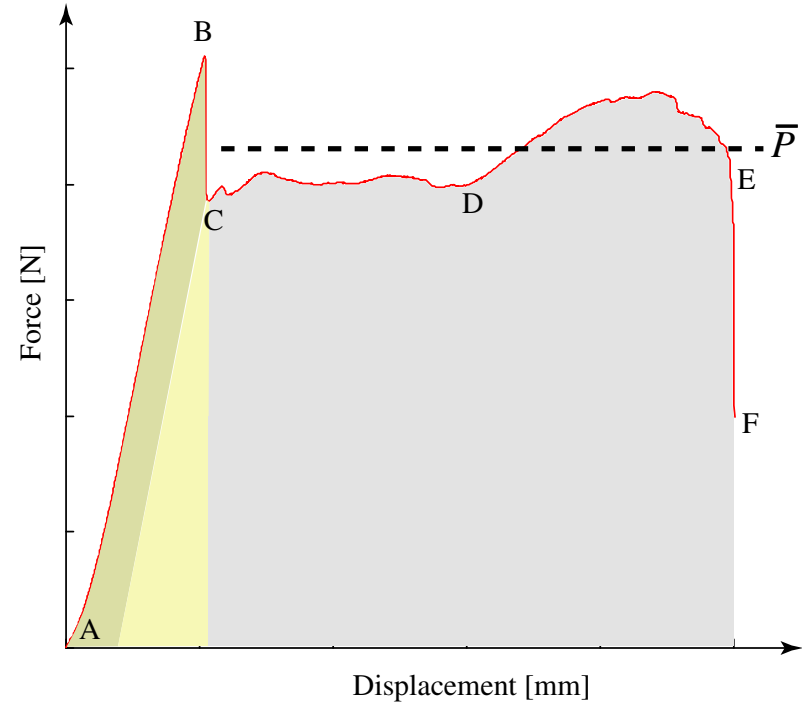

Fig. 8. A typical force-displacement curve from the "short beam test" (ASTM D2344). (A-F) denotes different deformation/damage stages of the test during quasi-static loading. $\bar{P}$ is the average residual force after the onset of initial damage.

structure which, along with the residual strength, reveals the postdamage behavior. Consequently, the average sustained force after initial damage, $\bar{P}$, can be used as a measure of the residual strength of the structure after initial damage.

Fig. 9 summarizes the normalized residual strength from the "short beam test" (ASTM 2344) for all structures, where the residual strength is normalized by the corresponding maximum force $\left(P_{B}\right)$ for each individual configuration. The bio-inspired structures demonstrate a remarkable increase of residual strength over the baseline structure (Fig. 9), especially those with the smaller fiber rotation; specifically, the single helicoidal mid-plane symmetric (SHMS) and single helicoidal (SH) structures (83\% for the SH and $56 \%$ for the SHMS). The double helicoidal (DH) structure shows a significant but smaller improvement over the baseline structure (23\% increase).

These results suggest that with the same parent materials and structural size, the bio-inspired structures (particularly the $\mathrm{SH}$ and SHMS) are able to retain significantly higher residual strength

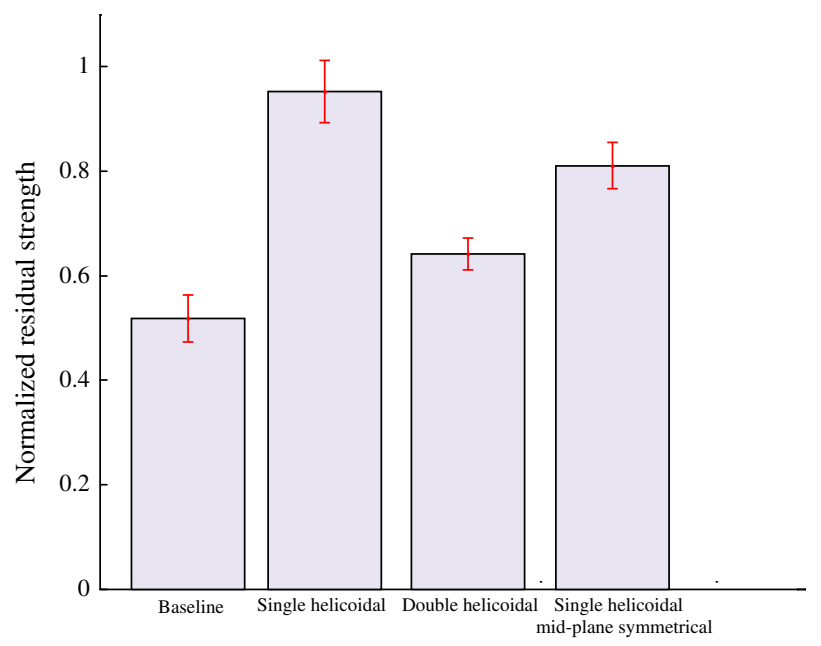

Fig. 9. Normalized residual strength of laminated composites with selected structures. The error bars indicate the standard deviation of the result within each group. 
( $83 \%$ for the SH and 56\% for the SHMS), after damage initiation and before final failure. Thus, the bio-inspired composites provide considerable improvement in damage resistance behavior over the baseline structure. This result agrees with the findings of other researchers [37-39], who argued that a smaller change of fiber orientation in the stacking sequence may increase the critical energy release rate of corresponding laminated composites and suppress the delamination propagation. The bio-inspired structural design (particularly SH and SHMS) confirms this principle by using a refined gradual lamina rotation to achieve a small and smooth fiber orientation change across the layer interfaces. As a result, it leads to a higher interlaminar fracture toughness for these laminates and consequently improved post-damage behavior. The marginal improvement of the $\mathrm{DH}$ laminate might possibly result from its "coarser" fiber rotation between layers, which ineffectively alleviates the stiffness mismatch across layer interfaces and as a result, gains only limited improvement over the baseline structure.

\section{Concluding remarks}

This study investigated the mechanical behavior of laminated composite structures designed and manufactured with bio-inspired lamina stacking sequences. The distinctive helicoidal structure observed in the exoskeletons of crustaceans (H. americanus and $C$. sapidus) and insect ( $P$. japonica) was incorporated into the bio-inspired structures using a glass fiber reinforced prepreg lamina. The helicoidal structure was characterized by a laminate stacking sequence in which each layer was rotated a small angle relative to its neighboring layer about their normal direction. Four configurations characterized by distinctive stacking sequences were developed: (1) a baseline structure (BL), which is widely used in industry as a quasi-isotropic structure; (2) a single helicoidal structure $(\mathrm{SH})$ with its stacking sequence directly replicated from the nature designed helicoidal structure; and two variations (3) a double helicoidal structure (DH); and (4) a single helicoidal mid-plane symmetric structure (SHMS). The last two configurations were developed to achieve mid-plane symmetry and avoid warping during cure.

The mechanical performance of the four structures was evaluated via standard test protocols (ASTM D790 and ASTM D2344) to quantify the flexural stiffness and strength, transverse shear modulus and strength, as well as residual strength.

The bio-inspired structure showed improved mechanical properties over the conventional baseline structure. In addition, superior residual strength under static load was observed from the bio-inspired composites. The improvement was more significant when a smaller fiber rotation was used, such as the SH and SHMS structures. Also, the advantages of the bio-material system were maintained for the two variants where mid-plane symmetry was enforced.

The improvement in mechanical performance observed in the bio-inspired structure underscored the advantages of the helicoidal structure. Combined with practical manufacturing practices, such as mid-plane symmetry, the nature designed helicoidal structure possesses great potential in future practical applications.

\section{Acknowledgements}

The authors would like to thank Mr. Anthony Thiravong and Mr. John Thiravong at the Center for Composite Materials, University of Delaware, for their help in the composite sample processing and testing. The authors gratefully acknowledge support from the United States Army Research Office.

\section{Appendix A. Classic laminate theory}

A short summary of the "classic laminate theory" is presented here. This theory can now be found in many text books, and notations consistent with that of Reddy [29] are used in the following text.

The linear-elastic stiffness of an individual lamina can be expressed via the stiffness matrix, $\mathbf{Q}$ [29],

$\mathbf{Q}=\left[\begin{array}{cccccc}Q_{11} & Q_{12} & Q_{13} & 0 & 0 & 0 \\ Q_{12} & Q_{22} & Q_{23} & 0 & 0 & 0 \\ Q_{13} & Q_{23} & Q_{33} & 0 & 0 & 0 \\ 0 & 0 & 0 & Q_{44} & 0 & 0 \\ 0 & 0 & 0 & 0 & Q_{55} & 0 \\ 0 & 0 & 0 & 0 & 0 & Q_{66}\end{array}\right]$

with the components $Q_{i j}$ :

$Q_{11}=E_{1}\left(1-v_{23} v_{32}\right) / \Delta, \quad Q_{22}=E_{2}\left(1-v_{31} v_{13}\right) / \Delta$,

$Q_{33}=E_{3}\left(1-v_{12} v_{21}\right) / \Delta, \quad Q_{44}=G_{23}, Q_{55}=G_{13}, Q_{66}=G_{12}$,

$Q_{12}=E_{1}\left(v_{21}+v_{31} v_{23}\right) / \Delta, \quad Q_{13}=E_{1}\left(v_{31}+v_{21} v_{32}\right) / \Delta$,

$Q_{23}=E_{2}\left(v_{32}+v_{12} v_{31}\right) / \Delta, \quad \Delta=4-v_{12} v_{21}-v_{23} v_{32}-v_{31} v_{13}-2 v_{21} v_{32} v_{13}$,

$(\mathrm{A} 2) \leftarrow$

where $E_{1}, E_{2}$ and $E_{3}$ are Young's moduli along the lamina's principle axis; $G_{12}, G_{13}$ and $G_{23}$ are the shear moduli; and $v_{i j}(i, j=1,2,3)$ are Poison's ratios.

When the laminae's principle axes are not aligned with the global coordinate system in which is structure is described, then a coordinate transformation is necessary to express the elastic properties of laminae with various orientations within the global coordinate system. The transformed stiffness matrix $\overline{\mathbf{Q}}$ can be expressed in matrix form as

$\overline{\mathbf{Q}}=\mathbf{T}^{-1} \mathbf{Q T}$,

where $\mathbf{T}$ is the transformation matrix

$\mathbf{T}=\left[\begin{array}{cccccc}m^{2} & n^{2} & 0 & 0 & 0 & 2 m n \\ n^{2} & m^{2} & 0 & 0 & 0 & -2 m n \\ 0 & 0 & 1 & 0 & 0 & 0 \\ 0 & 0 & 0 & m & -n & 0 \\ 0 & 0 & 0 & n & m & 0 \\ -m n & m n & 0 & 0 & 0 & m^{2}-n^{2}\end{array}\right]$

with $m=\cos \theta, n=\sin \theta$, where $\theta$ denotes the lamina orientation with respect to the global coordinate system.

Finally, the mechanical properties governing the laminate constitutive behavior are given:

$A_{i j}=\sum_{k=1}^{N} \bar{Q}_{i j}\left(h_{k}-h_{k-1}\right)$,

$B_{i j}=\frac{1}{2} \sum_{k=1}^{N} \bar{Q}_{i j} \quad h_{k}^{2}-h_{k-1}^{2} \quad, i, j=4,2,6$

$D_{i j}=\frac{1}{3} \sum_{k=1}^{N} \bar{Q}_{i j}{ }_{k} h_{k}^{3}-h_{k-1}^{3}$,

$A_{l m}=\sum_{k=1}^{N}\left(\bar{Q}_{l m}\right)_{k}\left(h_{k}-h_{k-1}\right), \quad l, m=44,5$,

where $A_{i j}$ are extensional stiffnesses, including transverse shear components when $i, j=4,5, B_{i j}$ are bending-extension coupling stiffnesses and $D_{i j}$ are flexural stiffnesses. $N$ is the number of lamina, $h_{k}$ is the distance from mid-surface to the upper side of the $k$ th layer and $h_{k-1}$ is the distance to the lower side of the $k$ th layer. 


\section{Reference}

[1] Cheng L, Wang LY, Karlsson AM. J Mater Res 2008;23(11):2854-72.

[2] Cheng L, Wang LY, Karlsson AM. J Mater Res 2009;24(11):3253-67.

[3] Neville AC. Biology of the arthropod cuticle. 2nd ed. New York (US): SpringerVerlag; 1975.

[4] Vincent JFV. Compos Part A - Appl Sci Manuf 2002;33(10):1311-5.

[5] Barbakadze N, Enders S, Gorb S, Arzt E. J Exp Biol 2006;209(4):722-30.

[6] Bruet BJF, Song JH, Boyce MC, Ortiz C. Nat Mater 2008;7(9):748-56.

[7] Neville AC. Biology of fibrous composites; development beyond the cell membrane. Cambridge: Cambridge University Press; 1993.

[8] Raabe D, Sachs C, Romano P. Acta Mater 2005;53(15):4281-92.

[9] Vincent JFV. Symp Soc Exp Biol 1980;34:183-210.

[10] Weiner S, Addadi L. J Mater Chem 1997;7(5):689-702.

[11] Andersen SO. Annu Rev Entomol 1979;24:29-61.

[12] Raabe D, Romano P, Sachs C, Al-Sawalmih A, Brokmeier H, Yi S, et al. J Cryst Growth 2005;283(1-2):1-7.

[13] Schultz TD, Rankin MA. J Exp Biol 1985;117(July):87-110.

[14] Nishino T, Matsui R, Nakamae K. J Polym Sci Part B - Polym Phys 1999;37(11):1191-6.

[15] Xu W, Mulhern PJ, Blackford BL, Jericho MH, Templeton I. Scanning Microscopy 1994;8(3):499-506.

[16] Neville AC, Parry DAD, Woodheadgalloway J. J Cell Sci 1976;21(1):73-82.

[17] Bouligan Y. Tissue Cell 1972;4(2):189-217.

[18] Wegst UGK, Ashby MF. Philos Mag 2004;84(21):2167-81.

[19] Apichattrabrut T, Ravi-Chandar K. Mech Adv Mater Struct 2006;13(1):61-76.

[20] Compere P, Goffinet G. Tissue Cell 1987;19(6):859-75.

[21] Compere P, Goffinet G. Tissue Cell 1987;19(6):839-57.
22] Gunderson S, Schiavone R. JOM - J Miner Metals Mater Soc 1989;41(11):60-2.

[23] Zelazny B, Neville AC. J Insect Physiol 1972;18(11):2095-121.

[24] ASTM. Standard test method for flexural properties of unreinforced and reinforced plastics and electrical insulating materials. In: (ASTM) ASfTaM, editor. American Society for Testing and Materials; 2003.

[25] ASTM. Standard test method for short-beam strength of polymer matrix composite materials and their laminates. In: (ASTM) ASfTaM, editor. American Society for Testing and Materials; 2006.

[26] Kim KS, Hahn HT. Compos Sci Technol 1989;36(2):121-32.

[27] ASTM. Standard test methods for void content of reinforced plastics. In: (ASTM) ASfTaM, editor. American Society for Testing and Materials; 2003.

[28] Polymer matrix composites guidelines for characterization of structural materials. In: Defense USDo, editor. US Department of Defense; 2002.

[29] Reddy JN. Mechanics of laminated composite plates: theory and analysis. 2nd ed. New York, US: CRC Press; 1997.

[30] Vinson JR, Sierakowski RL. The behavior of structures composed of composite materials. 2nd ed. Dordrecht/Boston/London: Kluwer Academic Publishers; 2002.

[31] Timoshenko SP, Gere JP. Theory of elastic stability. 2nd ed. New York: McGraw-Hill; 1959.

[32] Hashin Z. J Appl Mech - Trans ASME 1980;47(2):329-34

[33] Matzenmiller A, Lubliner J, Taylor RL. Mech Mater 1995;20(2):125-52.

[34] Liu KS, Tsai SW. Compos Sci Technol 1998;58(7):1023-32.

[35] Han YM, Hahn HT. Compos Sci Technol 1989;35(4):377-97.

[36] Hashin Z. Eng Fract Mech 1986;25(5-6):771-8.

[37] Cantwell WJ, Morton J. Composites 1991;22(5):347-62.

[38] Liu DH. J Compos Mater 1988;22(7):674-92.

[39] Tao JX, Sun CT. J Compos Mater 1998;32(21):1933-47. 